\title{
DECAIMIENTO EXPONENCIAL PARA LA ECUACIÓN DE LA ONDA SEMILINEAL CON POTENCIAL Y AMORTIGUAMIENTO LOCALMENTE DISTRIBUIDO
}

\author{
Alfonso Pérez Salvatierra ${ }^{1}$, Victoriano Yauri Luque ${ }^{1}$, Rodolfo Gálvez Pérez ${ }^{1}$
}

RESUMEN.- Se estudia el decaimiento exponencial de la ecuación de onda semilineal con potencial y amortiguamiento localmente distribuido, empleando el Principio de la Continuación Única, resultado estudiado por Ruíz [9] y aplicado a problemas de comportamientos asintóticos por Zuazua [10].

PALABRAS CLAVE: Decaimiento exponencial, Amortiguación local, Ecuación de onda semilineal, Principio de Continuación única.

\section{EXPONENCIAL DECAY FOR THE SEMILINEAR WAVE EQUATION WITH POTENTIAL AND LOCAL DISTRIBUTED DAMIPING}

\begin{abstract}
In this notes we shall study the exponential decay for the semilinear wave equation with potential and locally distributed damping, using the unique continuation principle, result studied by Ruíz [9] applied to problems of asymptotic behavior by Zuazua [10].
\end{abstract}

KEYWORDS.- Exponential decay, Locally damping, Semilinear wave equation, Unique continuation principle.

\section{INTRODUCCIÓN}

En esta sección plantearemos las hipótesis y algunos resultados necesarios para el desarrollo del presente trabajo.

H1. $\Omega \subset R^{\prime \prime}$ es un abierto, acotado $(n \geq 1), \Gamma=\partial \Omega$ frontera de clase $C^{2}$

H2. $W(x) \in L^{p}(\Omega), W \geq 0$ c.s. en $\Omega$ con $p=2$ sí $n=1$ y

$p>n$ para el caso en que $n \geq 2$.

H3. $a(x) \in L^{\infty}(\Omega), a(x) \geq a_{0}>0$ c.s. en $\omega$, donde $\omega \subseteq \Omega$ es una vecindad de $\Gamma=\partial \Omega$.

y $a(x)=0$ en $\Omega \backslash \omega$

H4. $f$ es una función semilineal que satisface:

i. ) $f(s) s \geq 0, \forall s \in R$.

ii ) $\exists \delta>0: f(s) s \geq(2+\delta) F(s) ; \forall s \in R$.

iii) $f$ tiene la propiedad del crecimiento, es decir,

${ }^{1}$ Universidad Nacional Mayor de San Marcos. Facultad de Ciencias Matemáticas. 


$$
\begin{aligned}
& |f(x)-f(y)| \leq C\left[1+|x|^{p-1}+|y|^{p-1}\right]|x-y| ; \forall x, y \in R \quad \text { y algún } \\
& C>0, p>1 \text { con }(n-2) p \leq n .
\end{aligned}
$$

iv) $F(s)=\int_{0}^{s} f(z) d z \geq 0, \forall s \in R$ pues $f(s) s \geq 0 ; \forall s \in R$.

Con las condiciones H1 - H4 se estudiará el sistema siguiente

$$
(*) \mid \begin{array}{ll}
u_{t t}-\Delta u+W(x) u+f(u)+a(x) u_{t}=0, & \text { en } \Omega \times(0, \infty) \\
u=0 & , \text { sobre } \Gamma x(0, \infty), \\
u(0)=u_{0} ; u_{t}(0)=u_{t} & , \text { en } \Omega .
\end{array}
$$

con datos iniciales $u_{0} \in H_{0}^{1}(\Omega), u_{1} \in L^{2}(\Omega)$, donde existe una única solución débil de (*) en la clase, $u \in C\left([0, \infty) ; H_{0}^{1}(\Omega)\right) \cap C^{1}\left([0,+\infty) ; L^{2}(\Omega)\right)$.

Podemos ver, referencias con aplicaciones en la física en Komornik [3], Muñoz [6], Pérez [7].

Se define la energía del sistema $(*)$ como

$$
E(t)=\frac{1}{2}\left\{\left|u_{t}\right|^{2}+|\nabla u|^{2}\right\}+\int_{\Omega} F(u) d x
$$

El objeto del trabajo es conseguir la estimativa de la energía para un $T>0$ adecuado,

$$
E(T) \leq C \int_{0}^{T} \int_{\Omega} a(x)\left|u_{t}(x, t)\right|^{2} d x d t
$$

Combinando (1) con la identidad dada por

$$
E\left(t_{2}\right)-E\left(t_{1}\right)=-\int_{t_{1}}^{t_{2}} \int_{\Omega} a(x)\left|u_{1}(x, t)\right|^{2} d x d t,
$$

Utilizando la Sección 1 de Zuazua [10] y la propiedad de semigrupos se obtiene el decaimiento uniforme exponencial de la energía para las soluciones del problema (*).

\section{RESULTADOS PREVIOS}

El resultado de Ruíz [9] nos dice, que si $b \in L^{\infty}(\omega \times(0, T)), w \in H^{1}(\Omega \times(0, \mathrm{~T}))$ y $T>\operatorname{diam}(\Omega)$ tal que $w$ satisface:

$$
(* *) \mid \begin{array}{ccc}
\left.w_{t t}-\Delta w+b x, t\right)=0 & \text { en } & \Omega \times(0, T) ; \\
w=0 & \text { en } & \omega \times(0, T) ; \\
w=0 & \text { sobre } & \partial \Omega \times(0, T) .
\end{array}
$$

entonces $w \equiv 0$ en $\Omega$. 
Este resultado caracteriza el método de la continuación única, que consiste en que si $w$ es solución del sistema $\left.{ }^{* *}\right)$, el cual es cero en una vecindad $\omega \times(0, T) \subset \Omega \times(0, T)$ de la frontera, esto es suficiente para que $w$ sea cero en $\Omega$.

Dada la ecuación de la onda no homogénea:

$$
(\bar{*}) \mid \begin{aligned}
\vartheta^{\prime \prime}-\Delta \vartheta & =f \quad \text { en } & & Q=\Omega \times[0, T] \\
\vartheta(0) & =\vartheta_{0} & , & \vartheta^{\prime}(0)=\vartheta_{1} \text { en } \Omega \\
\vartheta & =0 & \text { sobre } & \Sigma=\Gamma x[0, T]
\end{aligned}
$$

se deduce el siguiente lema.

\section{Lema 1.}

Sea $\Omega$ un dominio acotado de $R^{n}$ con frontera $\Gamma$ de clase $C^{2}, q=\left(q_{k}\right)$ un campo vectorial de clase $\left[C^{1}(\bar{\Omega})\right]^{n}$; entonces, para toda solución $\vartheta$ de $(\bar{*})$, se tiene

$$
\begin{aligned}
\int_{\Omega}\left(\vartheta^{\prime \prime}-\Delta \vartheta\right) q \cdot \Delta \vartheta=-\frac{1}{2} \int_{\Sigma} q \cdot v\left|\frac{\partial \vartheta}{\partial v}\right|^{2} & +\left(\int_{\Omega} \vartheta^{\prime} q \cdot \nabla \vartheta\right]_{0}^{T}+ \\
& +\int_{\Omega} \operatorname{div}(q)\left[\left|\vartheta^{\prime}\right|^{2}-|\nabla \vartheta|^{2}\right]+\int_{\Omega} \frac{\partial q_{k}}{\partial x_{j}} \frac{\partial \vartheta}{\partial x_{k}} \frac{\partial \vartheta}{\partial x_{j}}
\end{aligned}
$$

Demostración. Ver Lions [5], Lema 3.7 , págs. 40 - 41.

A partir del Lema 1, podemos deducir el lema siguiente.

\section{Lema 2.}

Para u solución de (*) y con las hipótesis H1 - H4 dadas en la introducción, $q=\left(q_{k}\right) \in\left[W^{1, \infty}(\Omega)\right]^{n}$ se tiene:

$$
\begin{aligned}
\left(\int_{\Omega} u_{t} q \cdot \nabla u\right]_{0}^{T} & +\frac{1}{2} \int_{Q} \operatorname{div}(q)\left[\left|u_{t}\right|^{2}-|\nabla u|^{2}\right]+\int_{Q} \frac{\partial q_{k}}{\partial x_{j}} \frac{\partial u}{\partial x_{k}} \frac{\partial u}{\partial x_{j}} \\
& -\int_{Q} \operatorname{div}(q) F(u)+\int_{Q} a(x) u_{t} q \cdot \nabla u=\frac{1}{2} \int_{\Sigma} q \cdot v\left|\frac{\partial u}{\partial v}\right|^{2}-\int_{Q} w(x) u q \cdot \nabla u .
\end{aligned}
$$

Demostración. Multiplicando (*) por $q . \nabla u$ y por el lema 1 , se obtiene el resultado.

\section{Proposición 1.}

Con las mismas hipótesis del Lema 2 y la definición de la energía del sistema (*) se tiene: 
$T E(T) \leq \int_{0}^{T} E(t) \leq C\left\{\int_{0}^{T} \int_{\Omega} a(x)\left|u_{t}\right|^{2}+\int_{0}^{T} \int_{\Omega}|u|^{2}+\left|\left(\int_{\Omega} u_{t} h \cdot \nabla u\right]_{0}^{T}\right|+|\hat{x}|+|\hat{y}|\right\}$

donde $\hat{x}=\left(\int_{\Omega} u_{t} m \cdot \nabla u+\alpha u\left(u_{t}+\frac{a u}{2}\right)\right]_{0}^{T} ;\left(\int_{\Omega} \eta u\left(u_{t}+\frac{a u}{2}\right)\right]_{0}^{T}$

Demostración. De los Lemas 1 y 2 se deduce la proposición.

\section{Lema 3.}

Considerando la definición de la energía del sistema $\left(^{*}\right) ; \hat{x}$ e $\hat{y}$ en la Proposición 1 y las hipotesis $\mathrm{H} 3$ - $\mathrm{H} 4$ se obtiene,

$$
|\hat{x}|+|\hat{y}|+\left|\left(\int_{t} h \cdot \nabla u\right]_{0}^{T}\right| \leq C\left[2 E(T)+\int_{0}^{T} \int_{\Omega} a\left|u_{t}\right|^{2}\right] .
$$

Demostración. De la definición de $\hat{x} e \hat{y}$

$$
\begin{aligned}
|\hat{x}|+|\hat{y}|+\left|\left(\int_{t} h \cdot \nabla u\right]_{0}^{T}\right| & =\left|\left(\int_{\Omega} u_{t} m \cdot \nabla u+\alpha u\left(u_{t}+\frac{a u}{2}\right)\right]_{0}^{T}\right|+ \\
& \left.+\mid \int_{\Omega} \eta u\left(u_{t}+\frac{a u}{2}\right)\right]_{0}^{T}|+|\left(\int_{\Omega} u_{t} h \cdot \nabla u\right]_{0}^{T} \mid \\
& \leq\left|\int_{\Omega}\right|\left[u_{t}(T) m \cdot \nabla u(T)+\alpha u(T)\left(u_{t}(T)+\frac{a u(T)}{2}\right)\right] \mid+ \\
& +\int_{\Omega}\left|\eta u(T)\left(u^{\prime}(T)+\frac{a u(T)}{2}\right)\right|+ \\
& +\int_{\Omega}\left|u_{t}(T) h \cdot \nabla u(T)\right|+\int_{\Omega}\left|u_{t} m \cdot \nabla u_{0}+\alpha u_{0}\left(u_{1}+\frac{a u_{0}}{2}\right)\right| \\
& \int_{\Omega}\left|\eta u_{0}\left(u_{1}+\frac{a u_{0}}{2}\right)\right|+\int_{\Omega}\left|u_{t} h \cdot \nabla u\right| .
\end{aligned}
$$

Las acotaciones de los 3 primeros sumandos del lado derecho de la desigualdad dada en (3) se realizan de la misma forma que en los 3 últimos sumandos, por lo que acotaremos sólo los 3 últimos sumandos. Por lo tanto de (3) tenemos 


$$
\begin{aligned}
\int_{\Omega}\left|u_{1} m \cdot \nabla u_{0}+\alpha u_{0}\left(u_{1}+\frac{a u_{0}}{2}\right)\right| & \leq \int_{\Omega}\left|u_{1}\right||m|\left|\nabla u_{0}\right|+\int_{\Omega} \alpha\left|u_{0}\right|\left|u_{1}+\frac{a u_{0}}{2}\right| \\
& \leq R\left(x^{0}\right) \int_{\Omega}\left|u_{1}\right|\left|\nabla u_{0}\right|+\alpha \int_{\Omega}\left|u_{0}\right|\left|u_{1}\right|+\frac{\alpha}{2}\|a\|_{L^{\infty}} \int_{\Omega}\left|u_{0}\right|^{2} \\
& \leq \frac{R\left(x^{0}\right)}{2}\left(\int_{\Omega}\left|u_{t}\right|^{2}+\int_{\Omega}\left|\nabla u_{0}\right|^{2}\right)+\frac{\alpha}{2}\left(\int_{\Omega}\left|u_{0}\right|^{2}+\int_{\Omega}\left|\nabla u_{1}\right|^{2}\right)+ \\
& +\alpha \frac{\|a\|}{2} L^{\infty} C \int_{\Omega}\left|\nabla u_{0}\right|^{2} \leq C\left[\int_{\Omega}\left|u_{1}\right|^{2}+\int_{\Omega}\left|\nabla u_{0}\right|^{2}\right] .
\end{aligned}
$$

Debido a que $|\eta| \leq 1$

$$
\int_{\Omega}\left|\eta u_{0}\left(u_{1}+\frac{a u_{0}}{2}\right)\right| \leq \int_{\Omega}|\eta|\left|u_{0}\right|\left|u_{1}+\frac{a u_{0}}{2}\right| \leq \int_{\Omega}\left|u_{0}\right|\left|u_{1}\right|+\int_{\Omega} \frac{a\left|u_{0}\right|^{2}}{2} .
$$

En forma análoga a la acotación del primer sumando se obtiene la acotación de la desigualdad (4).

$$
\int_{\Omega}\left|\eta u_{0}\left(u_{1}+\frac{a u_{0}}{2}\right)\right| \leq C\left[\int_{\Omega}\left|u_{1}\right|^{2}+\int_{\Omega}\left|\nabla u_{0}\right|^{2}\right]
$$

Finalmente se tiene

$$
\int_{\Omega}\left|u_{1} h . \nabla u_{0}\right| \leq \int_{\Omega}\left|u_{1}\right||h|\left|\nabla u_{0}\right| \leq\|h\|_{\left(w^{1, \infty}\right)^{n}} \int_{\Omega}\left|u_{1}\right|\left|\nabla u_{0}\right| \leq C\left[\int_{\Omega}\left|u_{1}\right|^{2}+\int_{\Omega}\left|\nabla u_{0}\right|^{2}\right] .
$$

Luego de las tres acotaciones y por la condición iv dada en H4 obtenemos

$$
\begin{aligned}
\int_{\Omega}\left|u_{1} m \cdot \nabla u_{0}+\alpha u_{0}\left(u_{1}+\frac{a u_{0}}{2}\right)\right| & +\int_{\Omega}\left|\eta u_{0}\left(u_{1}+\frac{a u_{0}}{2}\right)\right|+\int_{\Omega}\left|u_{1} h \cdot \nabla u_{0}\right| \leq \\
& \leq C\left[\int_{\Omega}\left|u_{t}(T)\right|^{2}+\int_{\Omega}|\nabla u(T)|^{2}\right] \leq C E(T) .
\end{aligned}
$$

Por tanto, se tiene

$$
|\hat{x}|+|\hat{y}|+\left|\left(\int_{\Omega} u_{t} h \cdot \nabla u\right]_{0}^{T}\right| \leq C[E(T)+E(0)]
$$

Haciendo $t_{2}=T$ y $t_{1}=0$ en (2) se tiene

$$
E(T)-E(0)=-\int_{0}^{T} \int_{\Omega} a\left|u_{t}\right|^{2} \Rightarrow E(0)-E(T)=\int_{0}^{T} \int_{\Omega} a\left|u_{t}\right|^{2} .
$$


Luego,

$$
E(0)+E(T)=2 E(T)+\int_{0}^{T} \int_{\Omega} a\left|u_{t}\right|^{2}
$$

Reemplazando (6) en (5) tenemos

$$
|\hat{x}|+|\hat{y}|+\left|\left(\int_{\Omega} u_{t} h \cdot \nabla u\right]_{0}^{T}\right| \leq C\left[2 E(T)+\int_{0}^{T} \int_{\Omega} a\left|u_{t}\right|^{2}\right] .
$$

\section{Proposición 2.}

Con las hipótesis dadas en la Proposición 1 y en el Lema 3 resulta

$$
E(T) \leq K\left[\int_{0}^{T} \int_{\Omega} a(x)\left|u_{t}\right|^{2}+\int_{0}^{T} \int_{\Omega}|u|^{2}\right] \text {, donde } K \text { es una constante positiva . }
$$

Demostración. Aplicando el Lema 3 en la Proposición 1 se obtiene

$$
\begin{aligned}
& T E(T) \leq C\left[\int_{0}^{T} \int_{\Omega} a\left|u_{t}\right|^{2}+\int_{0}^{T} \int_{\Omega}|u|^{2}+2 E(T)+\int_{0}^{T} \int_{\Omega} a\left|u_{t}\right|^{2}\right] . \\
& (T-2 C) E(T) \leq K\left[\int_{0}^{T} \int_{\Omega} a\left|u_{t}\right|^{2}+\int_{0}^{T} \int_{\Omega}|u|^{2}\right] . \\
& E(T) \leq \frac{K}{(T-2 C)}\left[\int_{0}^{T} \int_{\Omega} a\left|u_{t}\right|^{2}+\int_{0}^{T} \int_{\Omega}|u|^{2}\right], T \geq 2 C .
\end{aligned}
$$

Para $T$ suficientemente grande tal que $T>1+2 C$ tenemos

$$
E(T) \leq K\left[\int_{0}^{T} \int_{\Omega} a(x)\left|u_{t}\right|^{2}+\int_{0}^{T} \int_{\Omega}|u|^{2}\right], T>2 C
$$

\section{Proposición 3.}

Bajo las hipótesis H1 - H4, se tiene que,

$$
\int_{0}^{T} \int_{\Omega}|u|^{2} \leq C \int_{0}^{T} \int_{\Omega} a\left|u_{t}\right|^{2}
$$

Demostración. Se demuestra por el absurdo, suponiendo lo contrario y usando el Principio de la Continuación única de Ruíz [9] dado por el sistema (**), llegando a una contradicción. Este procedimiento es idéntico al usado en el Teorema 2.1, en Zuazua [10]. 


\section{EL TEOREMA CENTRAL}

Sea $\Omega \subset R^{n}$ abierto, acotado con frontera de clase $C^{2}$. Asumamos $f \in C^{1}(R), a \in L_{+}^{\infty}(\Omega)$ y, $W(x)$ satisfaciendo H4, H3 y H2, respectivamente. Entonces, para toda solución $u$ de $\left(^{*}\right)$ existen constantes $C>0$ y $\gamma>0$; tal que,

$$
E(t) \leq C e^{-\gamma t}, \forall t \geq 0
$$

Demostración. Usando la Proposición 3 en la Proposición 2, $\exists C>0$, tal que

$$
E(T) \leq C_{0} \int_{0}^{T} \int_{\Omega} a\left|u_{t}\right|^{2} d x d t, C_{0}=\max \{K, C\}
$$

Se deduce que; para $t_{1}<t_{2}$ en la energía se obtiene

$$
E\left(t_{2}\right)-E\left(t_{1}\right) \leq-\int_{t_{1}}^{t_{2}} \int_{\Omega} a\left|u_{t}\right|^{2} .
$$

Luego, si $T=t_{2}$ y $t_{1}=0$ se tiene

$$
E(T)-E(0)=-\int_{0}^{T} \int_{\Omega} a\left|u_{t}\right|^{2}
$$

De (6) y (7)

$$
E(T) \leq \frac{C_{0}}{1+C_{0}} E(0)
$$

Definimos $\alpha=\frac{C_{0}}{1+C_{0}}$, donde resulta que $\alpha<1, T>0$. Luego por la propiedad de semigrupo, y dado que el problema está bien puesto su solución es representada por

$$
U(t)=\left(u, u_{t}\right)=S(t) U_{0} \quad \text { Con } \quad U_{0}=\left(u_{0}, u_{1}\right) \in H_{0}^{1}(\Omega) \times L^{2}(\Omega) .
$$

Además, $S(t)$ satisface $S(t+s)=S(t) S(s)$, entonces

$$
E(n T)=E\left(S(n T) U_{0}\right)=E\left(S^{n}(T) U_{0}\right) \leq \alpha^{\prime \prime} E(0), 0<\alpha<1 .
$$

Para $T_{0}>2 R$, fijo y $s \in R^{+}$cualesquiera, existen $q, r \in R^{+}$tales que $s=q T_{0}+r$, con $r \in\left[0, T_{0}[\right.$, de donde se tiene

$$
t=s-r=q T_{0}, \quad t \in R^{+} .
$$


Luego,

$$
E(t) \leq \alpha^{q} E(0)=\exp \left[-\frac{1}{T_{0}}\left(\ln \alpha^{-1}\right)\right] t E(0)
$$

Por lo tanto,

$$
E(t) \leq C e^{-\gamma^{t}}, \forall t \geq 0, \text { donde } \gamma=\frac{1}{T_{0}}\left(\ln \alpha^{-1}\right)>0, C=E(0)>0,
$$

\section{COMENTARIOS}

Existen muchos métodos para el estudio del decaimiento de una ecuación de onda, con condiciones iniciales y de frontera. Nosotros abordamos el estudio del sistema $\left({ }^{*}\right)$ gracias al gran resultado obtenido por Ruíz [9], el del Principio de la Continuación Única usando herramientas de inmersiones de Sobolev y el método indirecto (reducción al absurdo).

Cada termino que se agrega en la ecuación de la onda, tiene una interpretación física, además sirve como término disipativo para que las soluciones tengan un decaimiento exponencial, que es lo óptimo, puesto que también existe decaimiento polinomial.

Podemos citar algunos sistemas que modelan fenómenos físicos como:

\section{Sistemas Parcialmente Viscoelásticos.}

$$
\mid \begin{array}{ll}
u_{t t}-\Delta u+\int_{0}^{t} g(t-\tau) \operatorname{div}\{a(x) \Delta u(x, \tau)\} d t=0 & \text { en } \\
u=0 & \text { sobre } \Gamma \times] 0,+\infty[ \\
u(x, 0)=u_{0}(x), u_{t}(x, 0)=u_{1}(x) & \text { en } \Omega
\end{array}
$$

Para mayor información ver Pérez [7].

\section{Sistema Parcialmente Termoelástico (unidimensional).}

$$
\begin{aligned}
& \mid \begin{array}{ll}
u_{t t}-\beta u_{x x}+(a(x) \theta)_{x}=0 & \text { en }] 0, L[\times] 0,+\infty[ \\
\theta_{t}-\alpha \theta_{x x}+a(x) u_{x t}=0 & \text { en }] L_{1}, L[\times] 0,+\infty[ \\
\left.u(x, 0)=u_{0}(x), u_{t}(x, 0)=u_{1}(x), \theta(x, 0)=\theta(0), \text { en }\right] 0, L[
\end{array} \\
& \alpha, \beta \text { constantes. }
\end{aligned}
$$

Ver Muñoz - Bisogmin - Bisogmin [6]. Otros ejemplos pueden verse en Cabanillas [1] y Portillo - Muñoz [8].

Si el término disipativo $u_{t}$ no estuviera presente posiblemente la energía del sistema $\left(^{*}\right)$ para soluciones débiles no tendría un decaimiento exponencial.

Considerando $\Omega$ como el cuerpo afectado por el fenómeno Físico, teniendo en cuenta que la función $a(x) \in L^{\infty}(\Omega)$ que acompaña al término disipativo $u_{t}$ determina un amortiguamiento local del fenómeno físico reducido a una vecindad $\omega$ de $\Gamma=\partial \Omega$, lo cual es suficiente para que el comportamiento del fenómeno físico se dé en todo el cuerpo. 
Graficamente podemos mostrar la vecindad $\omega$ en la figura

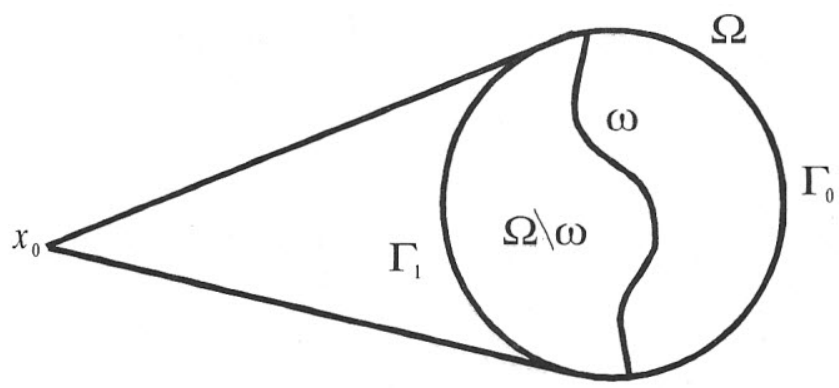

donde $\omega_{\varepsilon}=\omega=\bigcup_{x \in \Gamma}\left(B_{\varepsilon}(x) \cap \Omega\right), x_{0}$ punto fijo arbitrario y

$$
\begin{aligned}
& \Gamma_{0}=\{x \in \Gamma / m(x), v>0\} \\
& \Gamma_{1}=\{x \in \Gamma / m(x), v<0\} \\
& m(x)=x-x_{0} .
\end{aligned}
$$

\section{CONCLUSIÓN}

El presente trabajo estudia el fenómeno físico afectado solo en una parte de la frontera del cuerpo, lo que es suficiente para ser afectado sobre todo el cuerpo. Estos estudios en la actualidad son fuertemente aplicados en las diferentes ramas de la ciencia, tiene sus principios por los años 1990, a través de los artículos de Haraux [2], Komornik - Zuazua [4].

En los artículos recientes se viene trabajando con variación en la frontera incorporando una memoria.

\section{REFERENCIAS BIBLIOGRÁFICAS}

[1] Cabanillas, Eugenio, "Estabilización de la energía para una ecuación de Kirchdoff con disipación Localizada". Proyecto Inv. Inst. Matemática 2004.

[2] Haraux, A., "Stabilization of trayectories for some weakly damped hyperbolic equations", J. Differential Equation 59, 145 - 154 (1985).

[3] Komornik V., "Exact Controllability in Short Time for the Wave Equation". Ann. Inst. Poincaré, Analyce Nonlinéaire, pág. 153 - 164 (1989).

[4] Komornik V., and Zuazua E., "Stabilizatión frontiére de équation des ondes: Une Méthode directe". C. R. Acad. Sci. Paris 305605 - 608 (1987).

[5] Lions J. L., "Controlabilité exacte, perturbations et stabilisations de systémes Distribués". Tome 1. Contrabilité Exacte, RMA 8, Masson (1988).

[6] Muñoz R. J. Bisognin.V. - Bisognin E., "Exponential decay to partially thermoelastic, materials". LNCC, RJ. Brasil No 23/98 (1998).

[7] Peréz Alfonso, "Decaimento de soluções de equações Parcialmente Viscoelásticas". Tesis Doctorado UFRJ Brasil (1997).

[8] Portillo, Higinio - Múñoz, Jaime, "Sobre um problema do contato unidimensional de Ondas elásticas localmente Amortecidas". SBA 45 Seminario Brasileiro de Análise (1997).

[9] Ruíz A., "Unique continuation for weak solution of the wave equation plus a potencial". Jornal Math pure Applicada 71, pág. 455 - 467 (1992).

[10] Zuazua, Enrike, "Exponential decay for the semilinear wave equation with locally distributed damping". Comm. PDE 15, pp 205 - 235 (1990). 